\title{
Scanning Probe Microscopy of Atoms and Molecules on Insulating Films: From Imaging to Molecular Manipulation
}

\author{
Gerhard Meyer ${ }^{\star a}$, Leo Gross ${ }^{a}$, Fabian Mohnª, and Jascha Repp
}

\begin{abstract}
Scanning tunneling microscopy (STM) and atomic force microscopy (AFM) of single atoms and molecules on ultrathin insulating films have led to a wealth of novel observations and insights. Based on the reduced electronic coupling to the metallic substrate, these techniques allow the charge state of individual atoms to be controlled, orbitals of individual molecules to be imaged and metal-molecule complexes to be built up. Near-contact AFM adds the unique capabilities of imaging and probing the chemical structure of single molecules with atomic resolution. With the help of atomic/molecular manipulation techniques, chemical binding processes and molecular switches can be studied in detail.
\end{abstract}

Keywords: Atomic force microscopy - Insulating film · Molecular electronics · Scanning tunneling microscopy · Single molecule

\section{Introduction}

Scanning tunneling microscopy (STM) and atomic force microscopy (AFM) have the unique capability of enabling the study of single adsorbates at the atomic scale. Moreover, they can be used as a tool for atomic/molecular manipulation, ${ }^{[1,2]}$ i.e. for modifying surface structures atom by atom. Besides simple lateral displacement and reversible transfer of adsorbates between surface and probe tip, manipulation can also be used to control the properties of single atoms/molecules, for example by changing a molecular conformation, or by performing single molecule chemistry. ${ }^{[3]}$ An important step in this context was the development of low-temperature scanning probe microscopes, which operate at temperatures of liquid helium and even below. In this way, the mobility of weakly bound individual atoms and molecules can be suppressed. In addition, some other instrumental conditions are also improved

Correspondence: Dr. G. Meyer

alBM Research - Zurich

Säumerstr. 4

$\mathrm{CH}-8803$ Rüschlikon

Tel.: +41447248407

Fax: + 41447248958

E-mail: gme@zurich.ibm.com

bUniversität Regensburg

Institut für Experimentelle u. Angewandte Physik

D-93040 Regensburg, Germany at low temperature, such as the mechanical drift in the microscope and the surface contamination by adsorption from the gas phase. This way structures can be built up with atomic-scale precision to investigate particular physical phenomena under idealized and well-known conditions.

In which form these manipulation processes are effective and what types of manipulation can be applied depends very strongly on the substrate material. Accordingly, many investigations were performed on flat inert metal surfaces, such as copper, silver and gold, because their low diffusion barrier is ideal for lateral manipulation and hence for the construction of nanostructures. ${ }^{[4]}$ In contrast, on semiconductor surfaces, primarily electron-induced processes are used ${ }^{[5-7]}$ because of the stronger and more directional covalent bonding. Insulator surfaces cannot be directly investigated with STM, but are accessible in the form of ultrathin films on a metallic or semiconducting substrate (Fig. 1). Films of a thickness of up to $1 \mathrm{~nm}$ provide a sufficiently thin tunnel barrier, so that one can work at tunneling currents in the $\mathrm{pA}$ or $\mathrm{fA}$ range. The most important aspect of insulating films is the reduced electronic coupling of the adsorbate to the underlying metal substrate. In such a tunneling junction, the discrete electronic structure of the adsorbate is maintained, and one can tunnel resonantly through the discrete states of the adsorbate (Fig. 2). In this resonant tunneling process, the tunnel electron resides much longer on the adsorbate than in the case of adsorbates on metal surfaces, so that manipulation processes based on electron-phonon/vibron coupling are very efficient.

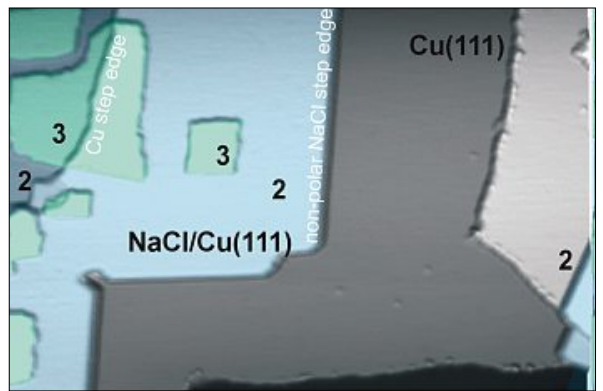

Fig. 1. $\mathrm{NaCl}$ island on $\mathrm{Cu}(111)$. The numbers indicate the $\mathrm{NaCl}$ film thicknesses in atomic layers. The island starts with a double layer and has straight nonpolar step edges. Substrate defect steps are smoothly overgrown. $(210 \mathrm{~nm} \times 250 \mathrm{~nm} ; 588 \mathrm{mV} ; 0.17 \mathrm{nA})$

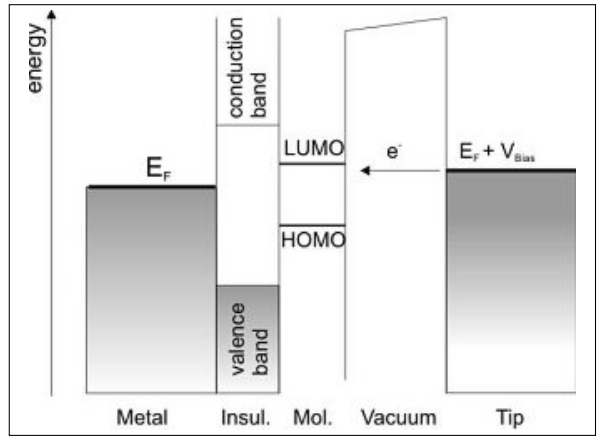

Fig. 2. Schematic model of a double-barrier tunneling junction. The molecule is separated from the STM tip apex by the vacuum barrier and from the metal substrate by the ultrathin insulating film.

\section{Controlling the Charge State of Metal Atoms}

An interesting manipulation process that is only possible in the presence of an 
insulating film is the control of the charge state of adsorbates, as was first demonstrated for single $\mathrm{Au}$ atoms on $\mathrm{NaCl} / \mathrm{Cu}(111){ }^{\left[{ }^{8]}\right.}$ By positioning the STM tip above a Au adatom and then applying a voltage pulse, the adatom can be reversibly switched between its neutral and its negatively charged state. Most importantly, both states are stable: an additional charge will remain on the adsorbate until it is removed again by a voltage pulse of reversed sign.

After deposition on $\mathrm{NaCl}(100) /$ $\mathrm{Cu}(111)$, individual $\mathrm{Au}$ adatoms are imaged as protrusions. The apparent height is in the range of 2.0-2.5 $\AA$ for a tunneling current of $I=10-200$ pA (Fig. 3). The adsorption site was determined to be on top of the $\mathrm{Cl}$ anion, both directly from atomically resolved STM images of the $\mathrm{Cl}$ anions as well as indirectly from the adatom position with respect to artificially created $\mathrm{Cl}$ vacancies. To determine the charge state, two approaches were used. One employs AFM and will be discussed later, whereas the other is based on the scattering of interface-state electrons by charged adsorbates. On $\mathrm{Cu}(111)$ the well-known surface-state band survives $\mathrm{NaCl}$ adsorption and forms an interface state band that is confined to the insulator/metal interface. ${ }^{[9]}$ In STM images, the interface state manifests itself as a typical standing-wave pattern ${ }^{[10]}$ arising from the scattering of electrons. The absence of scattering at the Au adatoms indicates that the Au adatoms remain neutral upon adsorption, and we will thus refer to them as $\mathrm{Au}^{0}$ in the following. The charge state manipulation of $\mathrm{Au}$ adatoms, which is shown in Fig. 3, works as follows. The tip is first positioned directly above a $\mathrm{Au}$ adatom. The feedback loop is switched off, and a positive voltage $\mathrm{V} \geq 0.6 \mathrm{~V}$ is applied. After a certain time $t$, which depends on the specific tunneling parameters, a sharp current drop by about a factor of 3 is observed. In subsequent images, the corresponding $\mathrm{Au}$ adatom appears differently, but is still at the same adsorption site. The manipulated $\mathrm{Au}$ adatom is imaged as a sombrero-like shape, with a protrusion that is about $0.5 \AA$ smaller than in the image of the original state and is surrounded by a depression. By applying a negative voltage pulse of about $-1 \mathrm{~V}$, the manipulated adatom can be switched back to its original state. Au adatoms in the manipulated state scatter the interface-state electrons, but no interface-state localization at the adatom can be observed. ${ }^{[8]}$ This indicates that the manipulated $\mathrm{Au}$ atom is negatively charged $\left(\mathrm{Au}^{-}\right)$. The states of the neutral and the negative Au adatom are both stable and must therefore be associated with two different geometric configurations of the adatom and the $\mathrm{NaCl}$ film. A simple electron transfer without lasting changes of the ion core positions would not be stable,
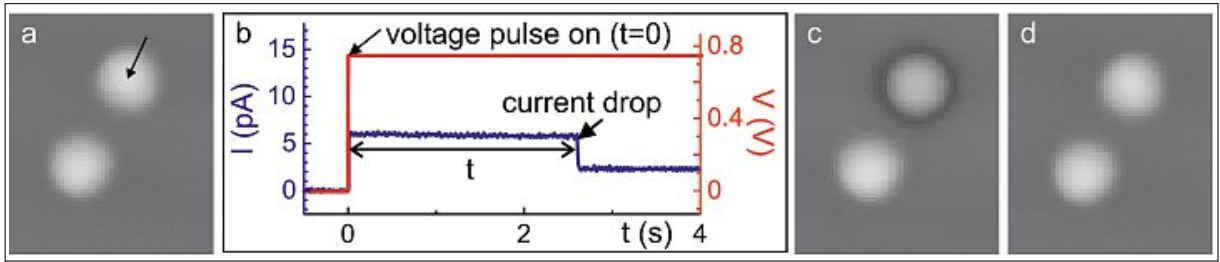

Fig. 3. Manipulation of the Au adatom state. After recording the image (a), the STM tip was positioned above one of the Au adatoms (arrow) and a positive voltage pulse was applied to the sample. (b) After a short time, a sudden decrease of the tunneling current can be observed. A subsequent STM image (c) shows that the manipulated Au adatom did not change its lateral position, but has a different appearance. By applying a negative voltage pulse, the manipulated adatom can be switched back to its initial state (d). (50 mV; 10 pA). Adapted from ref. [8].

because the electron residing in an excited state on the manipulated Au adatom would rapidly tunnel into the metal underneath.

Density functional theory (DFT) simulations ${ }^{[8]}$ provided deeper insight into the underlying principle of this experimentally observed charge bistability: In agreement with the experiments, the theoretical investigation found two different stable states for $\mathrm{Au}$ atoms on top of a $\mathrm{Cl}$ anion on the $\mathrm{NaCl}$ film on $\mathrm{Cu}(100)$, namely, a neutral and a negatively charged state. Whereas the $\mathrm{Au}$ atom in its neutral state leaves the ionic positions within the $\mathrm{NaCl}$ film relatively unperturbed, the negatively charged $\mathrm{Au}$ atom induces large ionic relaxations within the $\mathrm{NaCl}$ film. The $\mathrm{Cl}$ anion beneath the adatom is forced to move downward by $0.6 \AA$ and the surrounding $\mathrm{Na}$ cations are forced upward by $0.6 \AA$. This relaxation pattern creates an attractive potential for the additional charge on the Au adatom, which is further stabilized by the screening charge in the metal substrate and by the electronic polarization of the ionic layer. This shifts the $\mathrm{Au}(6 \mathrm{~s})$ state, which in a neutral $\mathrm{Au}$ adatom is singly occupied, downwards by approximately $1.0 \mathrm{eV}$, such that it is below the Fermi level and becomes doubly occupied in the negatively charged $\mathrm{Au}$ adatom.

The measured quantum yield, i.e. the probability of a manipulation event per tunneling electron, can reach values of order unity for voltage pulses of $\mathrm{V} \geq 1.4 \mathrm{~V}$. This extremely high yield is consistent with a capture of electrons tunneling resonantly into the negative ion resonance (NIR) state (the NIR is the negatively charged adsorbate, with the system in the same ionic configuration as for the neutral adsorbate). This suggests that, because of the presence of the insulator, the lifetime of the NIR state of the adatom is in the range of the ionic vibrational periods, which results in the capture of the tunneling electrons. An electron tunneling resonantly into the NIR state remains there for a sufficiently long time so that the adatom and its surrounding ions have time to relax, which leads to the NIR state moving below $E_{F}$ and the electron being captured. This model implies that the NIR is $1.4 \mathrm{eV}$ above the Fermi level or $2.6 \mathrm{eV}$ below the vacuum level, ${ }^{[11]}$ which is close to the electron affinity of an isolated $\mathrm{Au}$ atom of $2.3 \mathrm{eV}$ (below the vacuum level).

Whereas for $\mathrm{Au}$ adatoms charge bistability was observed, three charge states could be identified for $\mathrm{Ag}$ adatoms. $\mathrm{A} \mathrm{Ag}$ adatom can also be manipulated into a positively charged $\mathrm{Ag}^{+}$through hole tunneling induced by the application of a negative voltage pulse $(\mathrm{V} \geq-1.3 \mathrm{~V})$. In atomically resolved images, ${ }^{[12]} \mathrm{Ag}^{+}$was found at a site bridging two $\mathrm{Cl}$ anions, whereas $\mathrm{Ag}^{-}$and $\mathrm{Ag}^{0}$ were both found on top of $\mathrm{Cl}$ anions. Charge-state switching is not restricted to atoms, but can also be observed for single molecules, as was demonstrated for magnesium porphine and copper phthalocyanine molecules. ${ }^{[13]}$ Moreover, in the latter work, the spatial distribution of the additional charge was studied by the subtraction of STM images of the molecules in their various charge states. To realize different stable charge states, one electronic adsorbate state has to be sufficiently close to the Fermi level of the underlying substrate, as can often already be achieved by selecting a substrate with suitable work function. The ionic and electronic polarization can then lead to a shift of that state across the Fermi level of the substrate resulting in the stability of more than just one charge state. As the shift of the state due to polarization is stronger the more localized the charge is, the energy balance in the case of molecules is more subtle than in the case of metals. If the state is not shifted across the Fermi level, different charge states may still be accessible, but can only be realized in the presence of an electric field induced by the STM tip. ${ }^{[14]}$

AFM offers an alternative way to determine the charge-states of individual atoms. ${ }^{[15]}$ Charging of a $\mathrm{Au}$ atom by one electron charge, for example, increases the measured attractive force on the AFM tip by a few piconewtons. ${ }^{[15]}$ This is sufficient to clearly identify the charge state. Moreover, the local contact potential difference is shifted depending on the sign of the charge, allowing the discrimination of 
positively charged, neutral, and negatively charged atoms. The AFM employed in this study was based on a qPlus ${ }^{[16]}$ tuning-fork sensor operated at $5 \mathrm{~K}$ using very small oscillation amplitudes in the subangstrom regime.

\section{Imaging Molecular Orbitals with STM}

A remarkable result of the electronic decoupling of molecules on an ultrathin insulating film is the possibility to study the inherent electronic properties of these individual molecules. An important consequence is that one can image molecular orbitals using STM, or, more precisely, surfaces of constant electron density of the molecular orbitals. As these states have to be in the range of typical tunneling voltages, they often are the HOMO (highest occupied molecular orbital) and the LUMO (lowest unoccupied molecular orbital) only, i.e. the so-called frontier orbitals. ${ }^{[17]}$ In the case of molecules with highly delocalized electrons, such as in molecular wires, they can also include a series of orbitals. ${ }^{[18]}$ As an example, panels a and $b$ in Fig. 4 show the HOMO and the LUMO of pentacene. A comparison with theoretical calculations of the orbital density, i.e. the positions of the nodal planes of a free pentacene molecule, exhibits an almost perfect match. This means that the substrate in this case has only a minor effect on the orbital structure. As one probes these discrete states with tunneling electrons, one investigates the negative and positive ion resonances. These states are closely related to the ionization energy $(6.6 \mathrm{eV}$ for pentacene) and the electron affinity (1.35 $\mathrm{eV}$ for pentacene) of the free molecule, but shifted towards the Fermi level because of the electronic polarization of the $\mathrm{NaCl}$ film and the underlying metal, ${ }^{[17]}$ as discussed for the adatoms. Although this has a significant effect on the measured energy, the primary character of the orbitals, i.e. the symmetry and number of nodal planes, is maintained. Even for molecules directly adsorbed on metal surfaces, STM images with an intramolecular structure that resembles the LUMO have been obtained. ${ }^{[19-21]}$

In orbital imaging, the electronic structure of the tip plays a decisive role. For example, an STM tip functionalized with a specific molecule can significantly alter the appearance of an orbital. Whereas for a typical noble-metal terminated tip usually s-wave character predominates, terminating the STM tip with a CO molecule, for example, results in a clear p-wave character of the tip. ${ }^{[22]}$ This can be directly seen in images at voltages corresponding to the molecular resonances, in which the structure of the intramolecular corrugation is
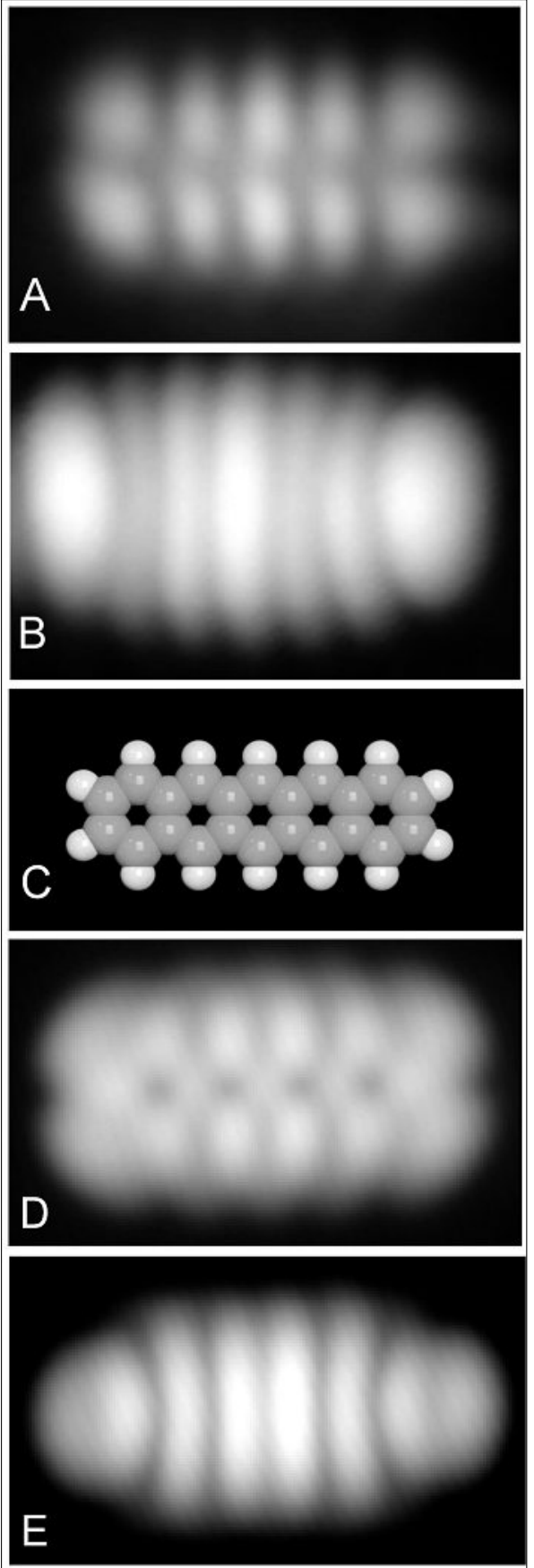

Fig. 4. STM images of pentacene (A) HOMO and (B) LUMO. (C) Model of the pentacene. (D) HOMO and (E) LUMO with CO-terminated tip. (A), (B), (C) adapted from ref. [17], (D), (E) adapted from ref. [22].

completely different. For a purely p-wave tip not the probability density of the wave function is imaged, i.e. the absolute square of the orbital wave function, but the square of the derivative of the wave function. ${ }^{[23]}$ In reality, the $\mathrm{CO}$ tip-termination is slightly more complex, as one also has to take into account that two degenerate $\pi$-orbitals contribute to the tunneling current. In general, when imaging orbitals of molecules with a CO tip, the tip's electronic structure can be best described by mixture of s-wave and $\mathrm{p}$-wave contributions. By comparing calculated s-wave and p-wave tip images with the mixed-tip images, it was found that the relative contributions of s- and pwave character depend not only on the tip itself but also on the lateral tip position. The relative $\mathrm{p}$-wave contribution of a $\mathrm{CO}$ tip is higher in regions in which the lateral gradient of the orbital becomes large. The influence of the s-wave tip is enhanced at outspread maxima of the orbital, where the amplitude is large but the gradient is small. In regions in which the p-wave contribution predominates, the gradient effect can be used to further enhance the spatial resolution in orbital imaging. ${ }^{[22]}$

\section{Resolving the Molecular Structure with AFM}

With STM the frontier orbitals of a molecule can be directly imaged, and AFM adds the unique capability to resolve the molecular structure with atomic resolution, as shown for example in Fig. 5 for a pentacene molecule adsorbed on a $\mathrm{NaCl}$ film. ${ }^{[24]}$ As can be seen in Fig. 5B and 5C, the five carbon hexagons are clearly resolved. Also the carbon-hydrogen bonds are imaged, indicating the positions of the hydrogen atoms within the pentacene molecule. In addition, each molecule is surrounded by a dark halo. The data represents the lateral dependence of the change in the frequency shift $\Delta f(x, y)$ of the tuningfork force sensor while the image is being recorded in the so-called constant-height mode, i.e. the tip is being scanned along a plane above the surface. As the oscillation amplitude of the tuning fork is only a few tens of picometers, the frequency shift is simply proportional to the gradient of the force between tip and sample. By recording the distance dependence of the force in each image pixel (3D force mapping, ${ }^{[25-27]}$ ) the interaction force and the energy can be determined by integration. ${ }^{[28]}$ Local maxima of $\Delta f(x, y)$ are observed above the edges of the hexagons, near the carbon atom positions, and minima above the centers of the carbon rings (hollow sites). To achieve this high resolution, the molecule is imaged under the regime of the short-range chemical forces. To be able to operate the non/near-contact AFM reliably in this regime, tip functionalization plays again a decisive role because with a normal metalterminated tip no intramolecular resolution could be obtained (Fig. 5A). To obtain the image shown in Fig. 5B, the tip was terminated with a single $\mathrm{CO}$ molecule. A similar resolution can be achieved by terminating the tip with a single chlorine atom, see Fig. 5C. The pentacene-modified tip (Fig. 5D), a termination used in STM to enhance orbital imaging, yields a completely different contrast than all the other tips investigated, indicating the strong influence of 


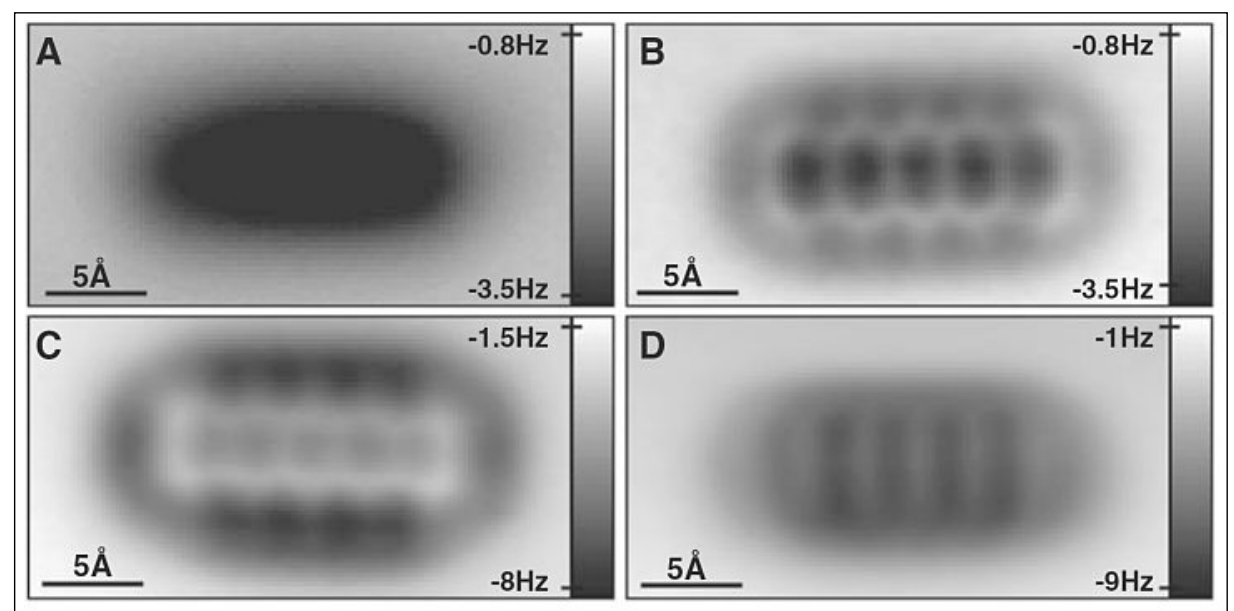

Fig. 5. Constant-height AFM images of pentacene $/ \mathrm{NaCl}(2 \mathrm{ML}) / \mathrm{Cu}(111)$ using different tip modifications. (A) Ag tip, $z=-0.7 \AA$, oscillation amplitude $A=0.6 \AA$; (B) CO tip, $z=+1.3 \AA$, $A=0.7 \AA$; (C) Cl tip, $z=-1.0 \AA, A=0.7 \AA$; (D) pentacene tip, $z=+0.6 \AA, A=0.5 \AA$. The $z$-values are given with respect to an STM set point of $I=2 \mathrm{pA}, V=200 \mathrm{mV}$ above the $\mathrm{NaCl}$ at positions next to the molecule. Adapted from ref. [24].

the tip modification. For CO termination, the $\mathrm{CO}$ molecule is transferred from the surface to the tip by vertical manipulation techniques and bonds to the tip, with the carbon bonding to the metal. Because the oxygen is then at the very end of the tip, the attractive interaction with the probed pentacene molecule is reduced, allowing the AFM to probe the short-range repulsive forces. In general, the lateral contrast of $\Delta f$ increases with decreasing tip height. At the height at which $\Delta f$ crossed zero, the highest contrast and lateral resolution with AFM were achieved. This is the region of maximal attractive forces. Decreasing the tip height further would result in instabilities and ultimately in the tip picking up the molecule.

To determine the origin of the high resolution, detailed theoretical calculations were made that took into account forces of three different physical origins, namely, electrostatic, van der Waals, and Pauli repulsive forces. By comparing their contributions to the overall force, it was found that the electrostatic forces are small $(\sim 10 \%)$. The electrostatic and van der Waals contributions to the force exhibit little lateral corrugation on the atomic scale and yield a diffuse attractive potential above the entire molecule, giving rise to the observed dark background surrounding the pentacene molecules in the $\Delta f$ maps. The atomic contrast is the result of the Pauli repulsion force, which becomes substantial when regions of high electron density overlap. These regions are at the atomic positions and at the $\mathrm{C}-\mathrm{C}$ (and also, but to a lesser extent, at the $\mathrm{C}-\mathrm{H}$ ) bonds in the pentacene molecule and are revealed for sufficiently small tip sample distances $(d \approx 5 \AA)$. Taking this a step further, it might become possible to extract details about intermolecular bonds, e.g. the bond order and length. One can foresee further developments, such as probing the reactivity of different molecular sites with respect to a known molecule or atom at the tip apex. Such investigations will yield detailed insight into chemical reactions and catalysis. Moreover, a combination with electrostatic force microscopy ${ }^{[15]}$ can be used to investigate single-electron transport in metal molecular systems on the atomic scale. Resolving structural details of molecules is not limited to AFM, as it has been observed that by using STM in the presence of hydrogen molecules the lateral resolution can be also strongly increased.[29]

\section{Molecular Switches}

Detailed orbital and structural information is extremely useful to investigate the functionality of singlemolecule devices. ${ }^{[30,31]}$ Here molecular switches stand out, as they have been intensively studied both in a two-terminal metal-molecule-metal geometry and by scanning probe techniques. ${ }^{[32]}$ In the following, we will discuss the properties of three different molecular switches based on bond breaking/formation in two metalmolecule complexes and on the currentinduced hydrogen tautomerization in naphthalocyanine molecules.

Orbital imaging allows one to directly examine the change of orbital structure induced by changes in the bond structure. As exemplary cases, we will discuss the bonding of a Au atom to a pentacene molecule and to a PTCDA (perylene-3,4,9,10-tetracarboxylic dianhydride) molecule. A Aupentacene complex is formed in several steps. ${ }^{[33]}$ First both reactants are positioned close to each other. Then a chemical bond is created between the two by means of

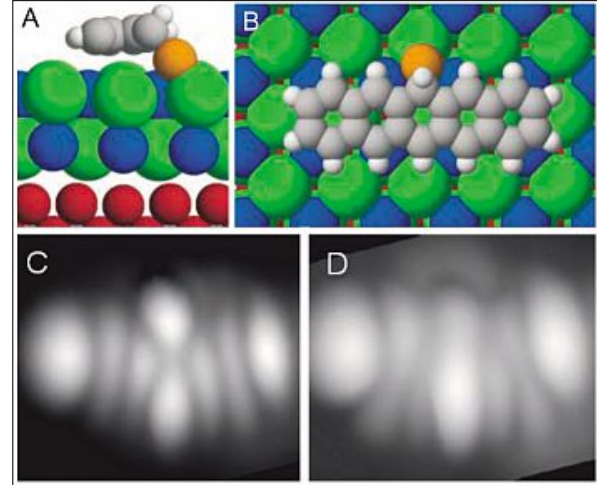

Fig. 6. (A), (B) Model of 6-Au-pentacene on an $\mathrm{NaCl}$ film. (C), (D) STM images of the SOMO with positive (C) and negative (D) sample voltage. Adapted from ref. [33].

nonthermal excitation using inelastic tunneling. Note that one can use the same process to break the bond again, i.e. the bonding process is reversible. The reversibility of the complex formation suggests that it is an addition reaction of the gold atom to one of the pentacene's aromatic rings and that it does not involve the substitution of a hydrogen atom. DFT calculations reveal that the bond is based on rehybridization of the bonding carbon atom from sp2 to sp3 (Fig. $6 \mathrm{~A}, \mathrm{~B})$, i.e. the carbon in the complex is bonded to four neighboring atoms instead of only three as in pentacene alone. This new bond will of course change the orbital structure. The most important experimental result is that orbital images at positive and negative voltages of the Au-pentacene complex look very similar, which means that tunneling into and out of the complex proceeds through the same orbital. The explanation lies in the fact that the $\mathrm{Au}-$ pentacene complex is a radical, i.e. it has an odd number of electrons. As pentacene has an even number and Au an odd number of electrons, the total electron number is odd (from the absence of interface scattering, one can experimentally determine that both the pentacene and the $\mathrm{Au}$-pentacene complex are neutral), and $\mathrm{Au}-$ pentacene has a singly occupied molecular orbital (SOMO). The locations of the nodal planes in the SOMO clearly differ from those in the HOMO and LUMO of the pentacene, which is evidence of the existence of a new bond. Analogously, also the orbital shape depends on which carbon atom the Au attaches to in the Au-pentacene complex. As the bonding site of the $\mathrm{Au}$ atom with respect to the pentacene can be controlled in the experiment, this can be viewed as orbital engineering.

The use of the Au-pentacene complex as a molecular switch is very limited because, in contrast to bond breaking, bond formation is very difficult to achieve and does not allow reliable switch operation. In this regard, a much better controlled and 

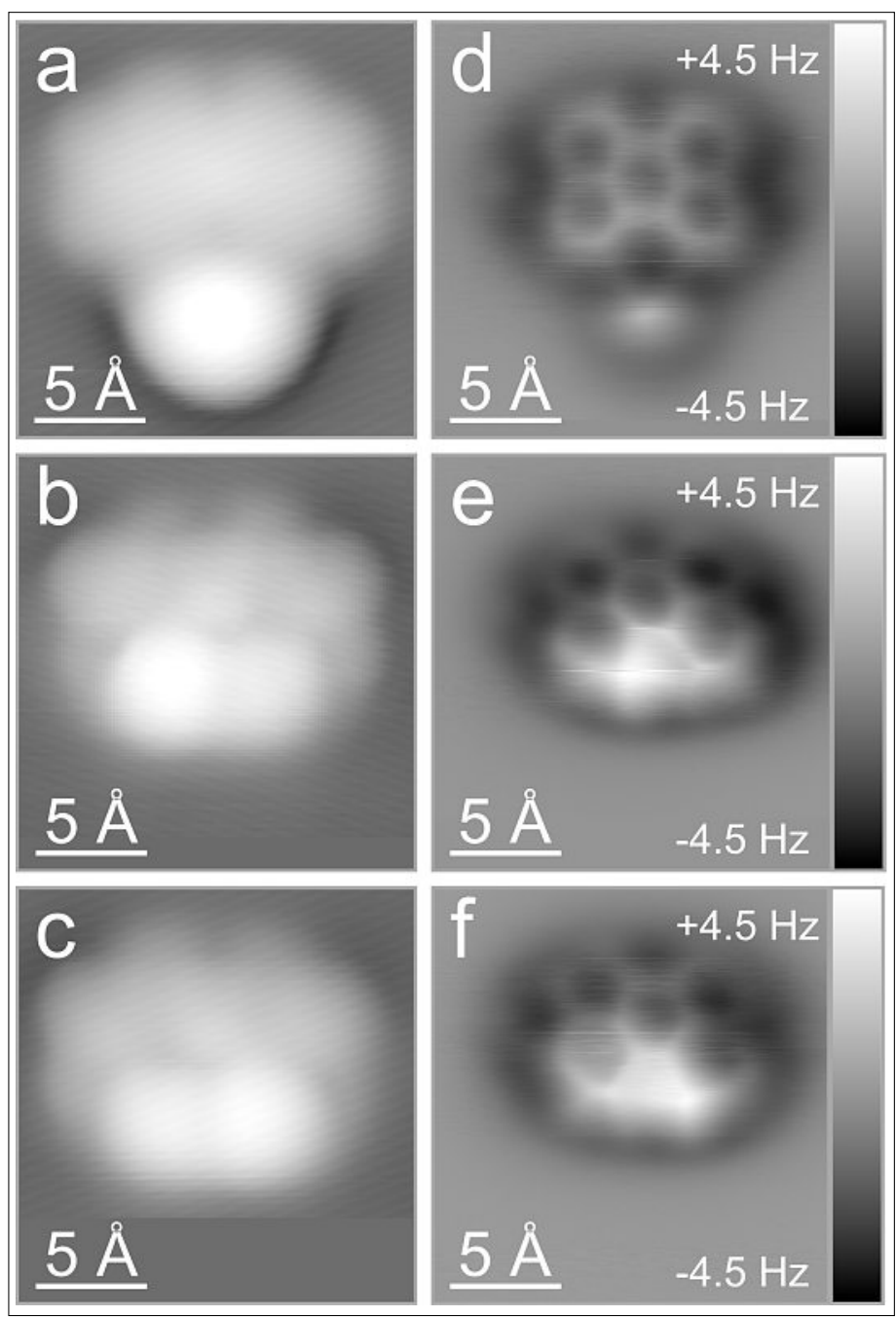

Fig. 7. Bond breaking/formation in $\mathrm{Au}-$ PTCDA. (a)-(c) STM images of Au-PTCDA in the (a) nonbonded and $(b, c)$ the bonded configuration (imaging parameters: $I=$ $3 \mathrm{pA}, V=0.2 \mathrm{~V}$ ). The tip was terminated with a $\mathrm{CO}$ molecule. (d)-(f) Corresponding constant-height AFM images. Imaging parameters: amplitude $A=0.4 \AA$, frequency $f=23,165 \mathrm{~Hz}$ and distance with respect to the STM set point above the substrate between $+0.8 \AA$ and $+1.0 \AA$. Adapted from ref. [34].

more reliable switching behavior was observed for Au-PTCDA. This metal-molecule complex has a number of characteristics that render it suitable as a molecular switch. First, whereas pentacene and $\mathrm{Au}-$ pentacene are neutral, PTCDA and $\mathrm{Au}-\mathrm{PTCDA}$ are both singly negatively charged. ${ }^{[34]}$ The reason lies in the greater electron affinity of the PTCDA molecule. The fact that the molecule and the complex are always charged plays a crucial role because the electrostatic interaction can be exploited to assist controlled bond formation. ${ }^{[34]}$ Bond making and breaking were controlled simply by applying voltage pulses of appropriate polarity, and did not require an exact tip movement or positioning over a particular part of the molecule. A simple model was suggested in which bond formation proceeds by temporarily removing a single electron charge from the PTCDA anion, reducing the Coulomb repulsion between the $\mathrm{Au}$ anion and the PTCDA molecule. Bond breaking proceeds by electron attachment, forming a doubly charged Au-PTCDA complex that is no longer stable and dissociates (Fig. 7). The electronic decoupling provided by the insulating $\mathrm{NaCl}$ film is crucial to ensure the stability of the different charge states. This mechanism seems to be much more reliable than bond activation by inelastic electron tunneling. Another relevant aspect of a molecular switch is the change in vertical conductance between the different states of the switch. For Au-PTCDA, the switching between the bonded and the nonbonded configuration was accompanied by a change in the tunneling current of about two orders of magnitude at certain bias voltages. This is because the energy and occupation of the orbitals change significantly upon bond formation/breaking, as the PTCDA anion has a singly occupied orbital, whereas the Au-PTCDA anion has completely occupied and unoccupied orbitals.

A very different kind of molecular switch can be realized with a naphthalocyanine molecule. ${ }^{[35]}$ This planar aromatic molecule has two hydrogen atoms in the center, and the change of their position is the basis of the switching process (Fig. 8). As it is energetically most favorable when the hydrogen atoms are opposing, i.e. in the trans configuration, by symmetry two (by $90^{\circ}$ mutually rotated) stable states of these two hydrogen atoms exist. Switching, i.e. the tunneling-induced hydrogen tautomerization, is achieved in the experiment by applying a voltage pulse. Because the orientation of the orbitals is tied to the symmetry of the molecule, this tautomerization can be directly seen in the twofold symmetric LUMO (Fig. 8). As the molecular geometry in the outer part of the molecule does not change, this switching is very reliable (tens of thousands of switching events could be recorded without any movement of the molecule), which is a prerequisite for coupling molecules laterally. In this way, the switching process could also be induced in adjacent molecules. ${ }^{[35]}$
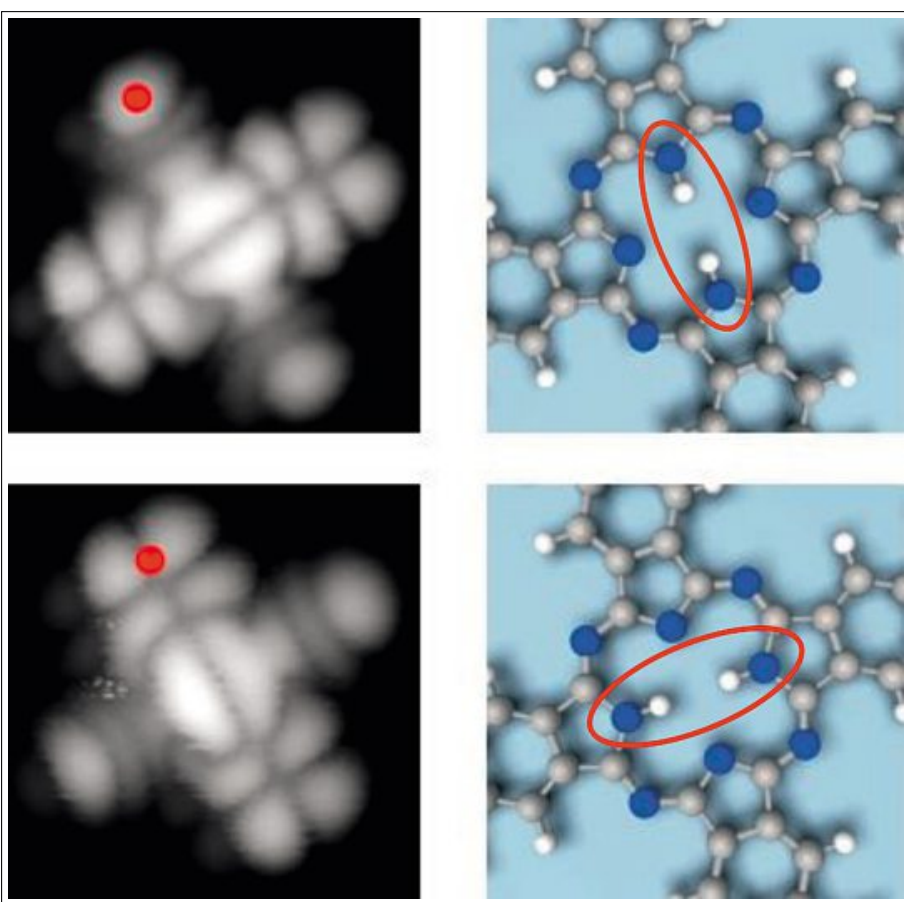

Fig. 8. Change in the orientation of the LUMO (left-hand side) of naphthalocyanine molecule by rearrangement of the two hydrogen atoms in the inner core of the molecule, as shown in the schematic model on the righthand side, representing the hydrogen tautomerization reaction responsible for the switching. Imaging parameters: $I=1$ $\mathrm{pA}$ and $V=0.65 \mathrm{~V}$. Adapted from ref. [35]. 


\section{Conclusion}

Scanning probe microscopy and atomic and molecular manipulation on ultrathin insulating films open up new possibilities for imaging and controlling matter on the atomic scale. Key techniques enabled thereby are the control of the charge state of single metal atoms and molecules, orbital imaging/engineering and the determination of the molecular structure. The STM-based processes are based on the weak electronic coupling to a conducting substrate and thus a significantly increased lifetime of the tunneling electron on the adsorbate. This gives rise to enhanced efficiency of bond-breaking and formation processes driven by inelastic tunneling, which, in combination with orbital imaging, opens up new possibilities in singlemolecule chemistry. A subject not mentioned in this review is the enhancement also of the spin lifetimes in single atoms on insulating films, which in combination with atomic manipulation has enabled a series of groundbreaking experiments using spin-polarized STM. [36-39]

As atomically perfect insulating films with varying thickness and of different materials are almost routinely grown, ${ }^{[40]}$ substrate-related properties can be conveniently studied and exploited. This provides deeper insight into and a better understanding of adsorbate-insulator interaction, a key aspect in surface and interface science, not only in the spatial but also in the temporal domain. ${ }^{[39]}$ With STM, such studies are limited to films of only a few atomic layers, but atomic force microscopy will allow their extension to thicker films without losing atomic resolution. ${ }^{[24,41]}$ This constitutes a first step in the investigation of new electronic transport phenomena in planar molecular networks, opening up new opportunities in single-molecule electronics. ${ }^{[42]}$

Received: November 6, 2011

[1] D. M. Eigler, E. K. Schweizer, Nature 1990, 344, 524.

[2] O. Custance, R. Perez, S. Morita, Nat. Nanotech. 2009, 4, 803 .

[3] W. Ho, J. Chem. Phys. 2002, 117, 11033.

[4] L. Bartels, G. Meyer, K.-H. Rieder, Phys. Rev. Lett. 1997, 79, 697.

[5] P. Avouris, Acc. Chem. Res. 1995, 28, 95.

[6] N. Lorente, R. Rurali, H. Tang, J. Phys. Cond. Mat. 2005, 17, S1049.

[7] A. J. Mayne, G. Dujardin, G. Comtet, D. Riedel, Chem. Rev. 2006, 106, 4355.

[8] J. Repp, G. Meyer, F. E. Olsson, M. Persson, Science 2004, 305, 493.

[9] J. Repp, G. Meyer, K.-H. Rieder, Phys. Rev. Lett. 2004, 92, 036803.

[10] M. F. Crommie, C. P. Lutz, D. M. Eigler, Science 1993, 262, 218

[11] R. Bennewitz, M. Bammerlin, M. Guggisberg, C. Loppacher, A. Baratoff, E. Meyer, H.-J. Güntherodt, Surf. Interface Anal. 1999, 27, 462.

[12] F. E. Olsson, S. Paavilainen, M. Persson, J. Repp, G. Meyer, Phys. Rev. Lett. 2007, 98, 176803.

[13] I. Swart, T. Sonnleitner, J. Repp, Nano Lett. 2011, 11, 1580; S. W. Wu, N. Ogawa, G. V. Nazin, W. Ho, J. Phys. Chem. C 2008, 112, 5241 .

[14] G. Mikaelian, N. Ogawa, X. W. Tu, W. Ho, J. Chem. Phys. 2006, 124, 131101.

[15] L. Gross, F. Mohn, P. Liljeroth, J. Repp, F. J. Giessibl, G. Meyer, Science 2009, 324, 1428.

[16] F. J. Giessibl, Rev. Mod. Phys. 2003, 75, 949.

[17] J. Repp, G. Meyer, S. M. Stojkovic, A. Gourdon, C. Joachim, Phys. Rev. Lett. 2005, 94, 026803.

[18] J. Repp, P. Liljeroth, G. Meyer, Nat. Phys. 2010, 6, 975.

[19] M. Böhringer, W.-D. Schneider, R. Berndt, Surf. Sci. 1998, 408, 72 .

[20] J. Lagoute, K. Kanisawa, S. Fölsch, Phys. Rev. B 2004, 70, 245415.
[21] W.-H. Soe, C. Manzano, A. De Sarkar, N. Chandrasekhar, C. Joachim, Phys. Rev. Lett 2009, 102, 176102

[22] L. Gross, N. Moll, F. Mohn, A. Curioni, G. Meyer, F. Hanke, M. Persson, Phys. Rev. Lett. 2011, 107, 086101.

[23] C. J. Chen, 'Introduction to Scanning Tunneling Microscopy', Oxford University Press, New York, 1993.

[24] L. Gross, F. Mohn, N. Moll, P. Liljeroth, G. Meyer, Science 2009, 325, 1110.

[25] H. Hölscher, S. M. Langkat, A. Schwarz, R. Wiesendanger, Appl. Phys. Lett. 2002, 81, 4428.

[26] M. Z. Baykara, T.C. Schwendemann, E. I. Altman, U. D. Schwarz, Adv. Mater. 2010, 22 2838.

[27] F. Mohn, L. Gross, G. Meyer, Appl. Phys. Lett 2011, 99, 053106

[28] J. E. Sader, S. P. Jarvis, Appl. Phys. Lett. 2004 $84,1801$.

[29] R. Temirov, S. Soubatch, O. Neucheva, A. C. Lassise, F. S. Tautz, New J. Phys. 2008, 10, 053012.

[30] C. Joachim, J. K. Gimzewski, A. Aviram, Nature 2000, 408, 541

[31] N. J. Tao, Nat. Nanotech. 2006, 1, 173.

[32] S. J. van der Molen, P. Liljeroth, J. Phys. Cond. Mat. 2010, 22, 133001

[33] J. Repp, G. Meyer, S. Paavilainen, F. E. Olsson, M. Persson, Science 2006, 312, 1196.

[34] F. Mohn, J. Repp, L. Gross, G. Meyer, M. S. Dyer, M. Persson, Phys. Rev. Lett, 2010, 105 , 266102

[35] P. Liljeroth, J. Repp, G. Meyer, Science 2007, 317,1203

[36] A. J. Heinrich, J. A. Gupta, C. P. Lutz, D. M Eigler, Science 2004, 306, 466.

[37] C. F. Hirjibehedin, C. P. Lutz, A. J. Heinrich, Science 2006, 312, 1021.

[38] C. F. Hirjibehedin, C.-Y. Lin, A. F. Otte, M Ternes, C. P. Lutz, B. A. Jones, A. J. Heinrich, Science 2007, 317, 1199.

[39] S. Loth, M. Etzkorn, C. P. Lutz, D. M. Eigler, A. J. Heinrich, Science 2010, 329, 1628.

[40] S. Schintke, S. Messerli, M. Pivetta, F. Patthey, L. Libioulle, M. Stengel, A. DeVita, W. D. Schneider, Phys. Rev. Lett. 2001, 87, 276801.

[41] L. Gross, F. Mohn, N. Moll, G. Meyer, R. Ebel, W. M. Abdel-Mageed, M. Jaspars, Nat. Chem 2010, 2, 821.

[42] M. Mayor, Chimia 2010, 64, 348. 